\title{
IMAGE ANALYSIS OF FUNCTIONAL MAGNETIC RESONANCE IMAGING
}

\author{
KaI-Hsiang Chuang ${ }^{1}$, Kou-Mou Huang ${ }^{2}$, Ping-Keung YiP ${ }^{3}$, \\ JYH-HORNG CHEN ${ }^{1}$, MING-JANG CHIU ${ }^{3}$
}

\author{
${ }^{1}$ Institute of Electrical Engineering, ${ }^{2}$ Department of Radiology, ${ }^{3}$ Department of Neurology, \\ National Taiwan University, Taipei, Taiwan
}

\section{INTRODUCTION}

Functional magnetic resonance imaging (fMRI) is an MRI technique, which produces images of activated brain regions by detecting the indirect effects of neural activity on regional cerebral blood volume (CBV), cerebral blood flow (CBF), and blood oxygenation [14]. It is a powerful tool for understanding the relationships between brain structure, function and pathology. Its non-invasive and non-radioactive nature adds on the potential and value as a diagnostic and research tool especially in fields such as studies of cognitive function requiring repetitive testing or critical evaluation of pre-surgical brain functions taking long-term monitoring.

Emerging in early 1990s, fMRI has been developed to be an imaging tool capable of sub-millimeter spatial resolution and sub-second temporal resolution. It identifies cortical areas active for a particular brain task and offers opportunity to rescan a subject in an easy and safe way. The advantages became evident when it was compared with the conventional radioactive functional imaging modalities, such as single photon emission computerized tomography (SPECT) or positron emission tomography (PET). The relative shortages of the latter modalities were long imaging time, low spatial resolution, and potential radiation hazards. Therefore, researchers and clinicians began to turn their attention to the development of fMRI technology and to acquaint themselves or to work with such new skill. Until the writing of this paper, there have been nearly 2,000 papers published according to Medline.

However, the functional signal changes during

Received: Oct. 12, 2001; accepted: Oct. 20. 20(01

Biomed Eng Appl Basis Comm, 2001 (Octoher); 13: 248-255 Correspondence: Dr. Ming-Jang Chiu, Department of Neurology, National Taiwan University Hospital, No. 7, Chung-Shan S. Rd., Taipei, 100, Taiwan

E-mail: chiu(a)ceg.mc.ntu.edu.tw brain activation in $\mathrm{fMRI}$ is very small, i.e., relative low signal-to-noise ratio (SNR) between activated and nonactivated states. Thus, post-processing is necessary to identify the location and signal of functional activation from the magnetic resonance (MR) images. The purpose of this article is to review and to compare different strategies for image post-processing. In addition, the principle and analysis procedures of $\mathrm{MMRI}$ will also be introduced.

\subsection{Principle of fMRI}

There are three major techniques for fMRI. In the first method, exogenous susceptibility contrast agent, such as intra-vascular paramagnetic contrast agent gadolinium diethylene-triamine-pentaacetic acid (GdDTPA), is applied to map the change of CBV following neural activity [5,6]. The second approach is the perfusion technique [7-9] which detects variations in apparent $\mathrm{T} 1$ values induced by changing flow during brain activation. The third one which exploiting the magnetic properties of oxygenated blood as a source of endogenous contrast, called blood oxygenation level dependent (BOLD) contrast $[1,2]$, to yield functional information is the most popular technique.

The underlying rationale for BOLD effect is based on the magnetism of red blood cell (RBC) $[10,11]$. When oxygen binds to the hemoglobin molecule in the $R B C$, the $R B C$ is diamagnetic, which property is similar to the surrounding tissues. After it releases oxygen, the molecule (deoxyhemoglobin) becomes paramagnetic, which will make perturbation in local magnetic field. This fluctuation in magnetic field facilitates intra-voxel dephasing of the MR signal due to water diffusion and/or field inhomogeneity. This phenomenon can be observed as decreased signal intensity in T2*- or T2-weighted MR images[12].

When neural activity occurs, the active transport of ions and neurotransmitters increases the glucose consumption, which in turn increase CBF to compensate the metabolic demand. However, a series of PET studies $[13,14]$ show that in contrast to the large stimulus-induced increase in CBF and glucose consumption, there is a very small increase in oxygen consumption. This uncoupling phenomenon leads to a large increase 
in the oxyhemoglobin content on the venous side of the capillary bed, producing a relative decrease in the fraction of deoxyhemoglobin in the tissue. Thus, the local susceptibility variation is reduced and the $\mathrm{T} 2{ }^{*}$ - or T2-weighted MRI signal intensity will be increased $[2,3]$. Because of the endogenous nature of this contrast agent, BOLD contrast-based MRI is currently the most widely used fMRI method for assessing brain neural activity.

To utilize this phenomenon, an fMRI experiment should consists of at least two states: one is for control and the other is for activation. During the activated state, the subject is instructed to perform some task, like finger tapping or reading. Usually, these states will be repeated for several times to increase SNR (Fig 1). In the mean time, a T2*-sensitive fast imaging sequence, like echo-planar imaging (EPI), will be performed to capture the temporal dynamics of the BOLD signal [15].

\section{THE ANALYSIS OF fMRI SIGNAL}

The analysis of fMRI data includes two major steps: preprocessing and analysis.

\subsection{Preprocessing}

In order to identify the small and sometimes variable signal change in the functional images, there are several preprocessing steps before going into statistical analysis of the functional changes.

\subsection{Realignment}

Movement-related variance induced by gross head motion in fMRI time-series represents one of the most serious confounds of analysis. Before going into analysis, head motion detection should be made to evaluate the quality of data. Two simple ways can be used. One is to measure temporal variation in the center-of-mass of the brain (Fig. 2). This method provides a global measure of the motion. The other method is to calculate the standard deviation of every pixel timecourses (Fig. 3). By mapping the standard deviation, the place with significant signal fluctuation can be scen.

When significant head motion is detected, image registration can be used to aligning all time-series images to the first image using a rigid body model, such as AIR (automated image registration, Fig. 4) [16]. However, movement-related effect in fMRI may be further confounded by that movement in earlier scans can affect signal in subsequent scans due to differential spin-excitation histories [17]. Therefore, simple realignment is necessary but incomplete for such correction. The adjustment may be furthered by correction based on an estimate from a moving average autoregression model of spin-excitation history effects.

\subsection{Spatial Normalization}

To implement a voxel-based analysis of imaging data, data from different subjects must derive from homologous parts of the brain. Spatial transformations are therefore applied that move and "wrap" the images that they all conform approximately to some idealized standard brain. The transformation of an image into a standard anatomical space, usually that described in the atlas of Talairach and Toumoux [18] as proposed by Fox et al [19].

\subsection{Spatial Smoothing}

There are several advantages of spatial smoothing. First, it generally increases SNR. The neuropsychological effects of interest are produced by homodynamic changes that are expressed over spatial scales of several millimeters, whereas noise usually has higher spatial frequencies. In $\mathrm{fMRI}$ the noise can be regarded as independent for each voxel and has therefore very high spatial frequency components. Second, it enhances statistical inference (Fig. 5). In order to smooth functional images we convolve the data with a smoothing kernel. The convolution with a Gaussian kernel conditions the data more closely to a Gaussian field model. This is important when we makes statistical inference. Finally, the inter-subject comparison or averaging will be only meaningful when homologies of the functional anatomy can be ensured.

\subsection{Image Enhancement and Background Canceling}

In order to remove the random noise and to maintain the boundary information while producing no additional artifact, the images can be filtered with an anisotropic filter[20]. The algorithm is shown as follows:

$$
\frac{\partial}{\partial t} I(\bar{x}, t)=\operatorname{div}(c(\bar{x}, t) \nabla I(\bar{x}, t))
$$

in which

$$
c(\bar{x}, t)=\exp \left(-\left(\frac{|\nabla I(\bar{x}, t)|}{\kappa}\right)^{2}\right)
$$

There are many black background pixels around MRI of the brain, which can be removed before the computation since they are not meaningful for signal calculation or classification. The threshold can be computed experimentally by one-tenth of the maximum pixel value of a MR image.

\subsection{Segmentation}

Image segmentation is very useful in separating gray matter, white matter, cerebrospinal fluid, blood vessels, and other brain structures. Segmentation methods usually utilize the differences in intensity distribution of different tissues. An unsupervised approach combines Kohonen self-organizing feature map and fuzzy c-means can classify brain into six different 
tissue using T1-weighted, T2-weighted and proton density weighted images. In fMRI study, segmenting these structures helps differentiating functional responses in gray matter from large vessels. Thus provides better spatial localization and quantification accuracy $[21]$.

\subsection{Analysis Methods}

The procedures used to find the extent and magnitude of response in the brain related to a stimulus or behavior task can be divided into three steps. First, data are analyzed to find the regions with MR signal changes temporally correlate with the experiment paradigm. Second, a threshold is used to discriminate the "inactive" brain regions (i.e., those with signal changes that are more consistent with noise) from the "active" regions. Finally, the results of the activation analysis are registered to high-resolution structural images, which are used to more accurately determine the brain structures involved in the activation task.

In the first step, there are two categories of methods: model-based and model-free. In model-based approaches, some postulation about functional signal change is made and is tested by hypothesis-driven statistical methods. This approach provides reliable analysis of the known functional responses. Thus it is most widely applied. Methods belonging to this category include: correlation analysis[22], t-test[23], general linear model[24], etc. Unlike traditional statistical analysis, in these methods a single $P$ value tells one nothing about regionally specific effects. Statistical inference about specific regional changes requires statistical parametric mapping. $P$ values pertain to different levels of inference, including: (i) the number of activated regions (i.e., number of clusters above some height and volume thresholds), (ii) the number of activated voxels comprising a particular region, and (iii) the $P$ value for each voxel within that region.

Although statistical methods are capable of identifying the functional responses from the MR images, they depend on some prior knowledge or assumption of the physiological response in brain activation. In many real-world conditions, however, the actual functional response is unpredictable or deviates from what we had anticipated. Then it is difficult to define the reference waveform or the activated and rest states. For example, in an eating study the response onset at hypothalamus is a few minutes later than the glucose intake and with quite different waveform[25]. In an olfactory study, the fMRI response shows significant faligue property and cannot be found by methods based on the model not considering this phenomenon[26]. Hence, the development of data-driven post-processing methods capable of identifying unknown response pattern becomes crucial. Among these techniques, independent component analysis (ICA) and temporal clustering are the most popular ones.

\section{MODEL-BASED METHODS}

\subsection{Direction Subtraction}

The simplest and most straightforward method is that we subtract the average signals during activation by the average signals during rest condition. This method is very sensitive to changes from all sources of signal fluctuation including unwanted noises and artifacts (Fig. 6). Therefore, due to its poor noise tolerance it is no longer applied either for clinical practice or for basic science research.

\subsection{Correlation Analysis}

Since the functional response follows the stimulus paradigm, the brain region with its MR signal timecourse that most correlates with the reference waveform derived from the paradigm should be the activated region (Fig. 7). To evaluate how correlate the time response function of fMRI data is with the reference function, the correlation coefficient (CC) between these waveforms will be calculated in a pixel-by-pixel manner (Fig. 5a). The result will be a two-dimensional correlation coefficient map $\mathrm{CC}_{(\mathrm{x}, \mathrm{y})}$ :

$$
C C_{(x, y)}=\frac{\sum_{i=1}^{N}\left[P_{(x, y)}(t)-\mu_{P(x, y)} \| R(t)-\mu_{R}\right]}{\sqrt{\sum_{i=1}^{N}\left(P_{(x, y)}(t)-\mu_{P(x, y)}\right)^{2}} \sqrt{\sum_{-A}^{N}\left(R(t)-\mu_{R}\right)^{2}}}
$$

where $P_{(\mathrm{x}, \mathrm{y})}(t)$ is the signal time-course at coordinate $(x, y)$ and time point $t ; \mu \mu_{x, y}$ represents the mean value of the time course at $P_{(\mathrm{x}, \mathrm{y})} ; R(t)$ is the reference function; $\mu_{\mathrm{R}}$ is the mean value of the $R(t)$; and $N$ is the number of scan. The simplest form of a reference waveform is a square-wave like function. Since the delectability is highly dependent on the reference waveform, a more elaborated method is to convolve the square-wave function with a idealized hemodynamic response function (Fig. 8).

In order to determine the level of significance, we can use $t$-distribution to compute the probability of type I error i.e., the probability of rejecting the null hypothesis when it is true. The $\mathrm{t}$-value is defined by:

$$
t=\frac{C C \sqrt{N-2}}{\sqrt{1-C C^{2}}}
$$

\subsection{Student t-Test}

Student t-test is used to evaluate whether two sample-means are statistically different or not. When applied to fMRI, it is used to examine whether the mean signal levels of a MR pixel time-course are significantly different between different activated states. It provides a probabilistic measure of the likelihood that an experimental observation was the result of ran- 


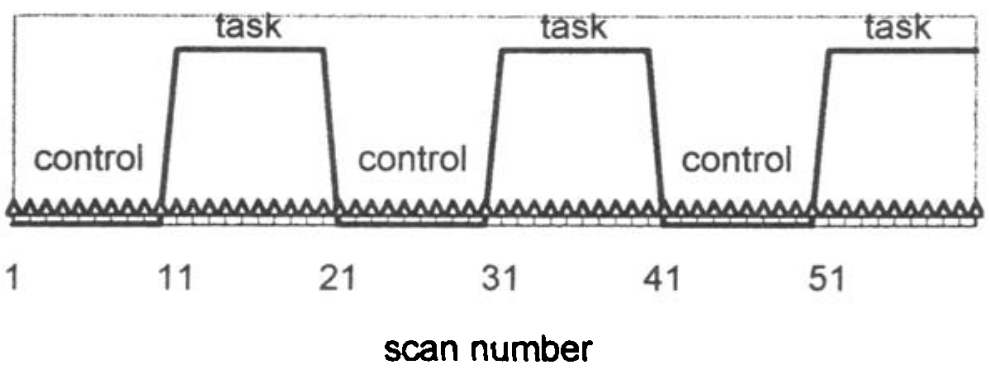

Fig. 1 A typical fMRI experiment with alternated control and task stimulating states. While

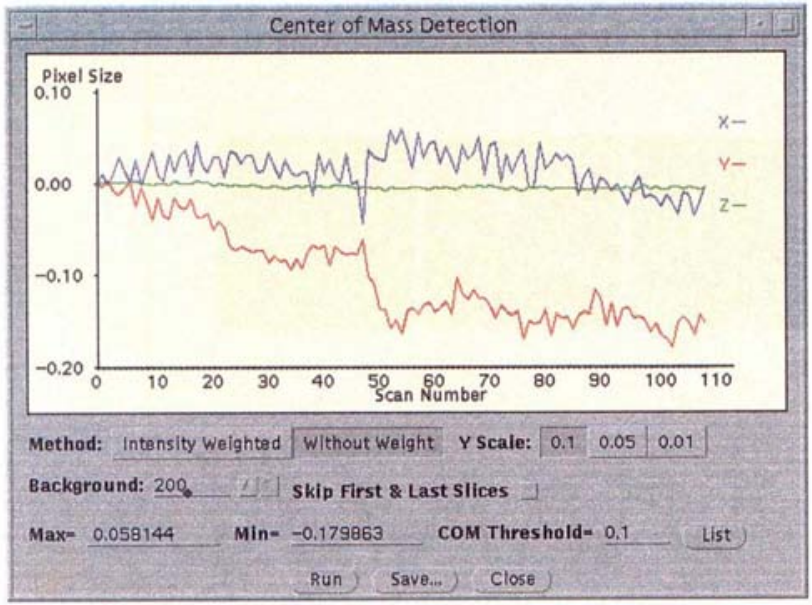

Fig. 2 Time course of the variation in center-ofmass of the brain. Displacement larger than onetenth of the size of a pixel is usually not acceptable.

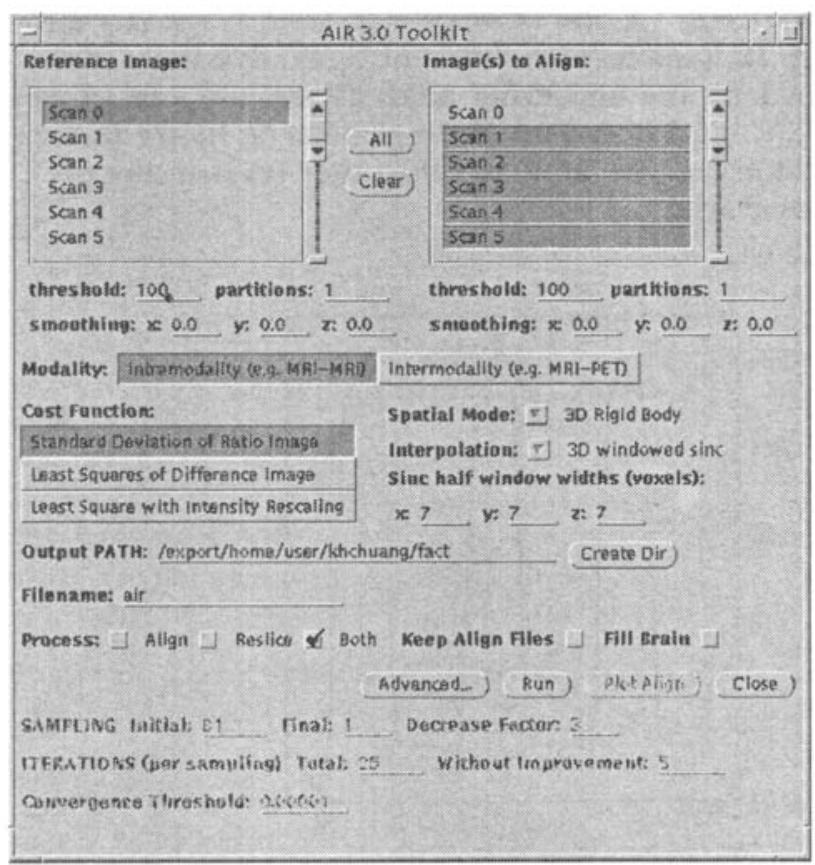

Fig 4. A graphical user interface (GUI) for automated image registration (AIR). For simplicity, the brain is usually assumed to be a rigid body.

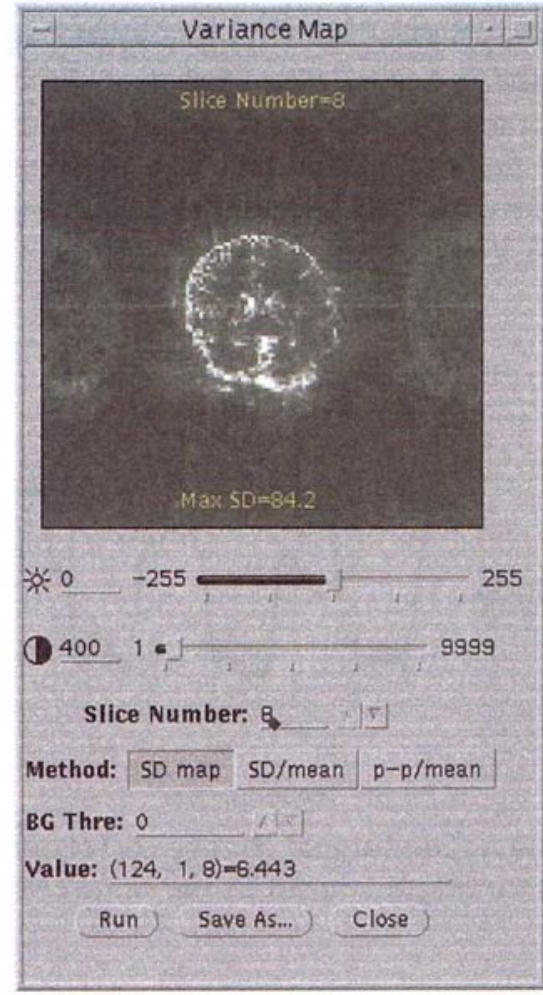

Fig. 3 The standard deviation map of an axial slice. The bright part in the map indicates that significant signal fluctuation appears on the edge, ventricles, and vessels.

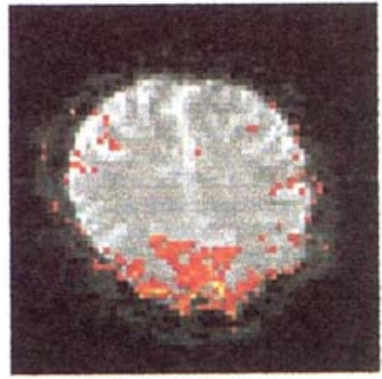

Fig. 6. Direct subtraction map of a visual fMRI study thresholded at signal change of $1 \%$. This map is very sensitive to signal variations. 

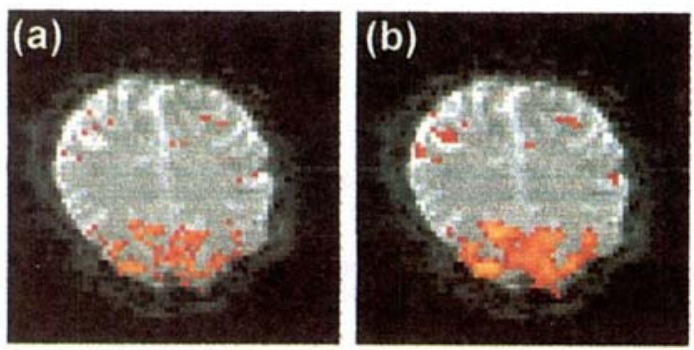

Fig. 5. Comparison of the visual stimulation activation map (a) before and (b) after spatial smoothing by a Gaussian kernel. The statistical significance is increased and the activated region is larger under the same threshold. The data was analyzed by correlation analysis with correlation coefficient thresholded at 0.35 .

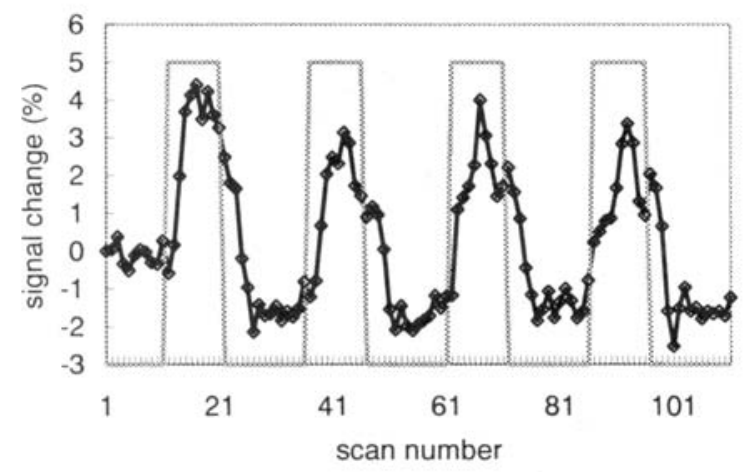

Fig. 7 Task-related signal change correlates with the stimulus paradigm (shown as the square-wave function).

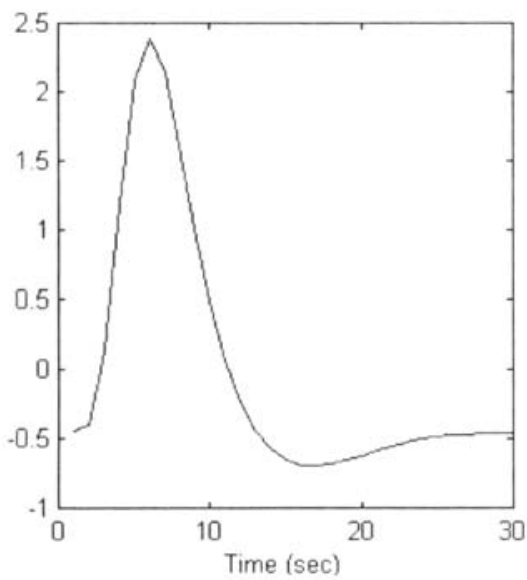

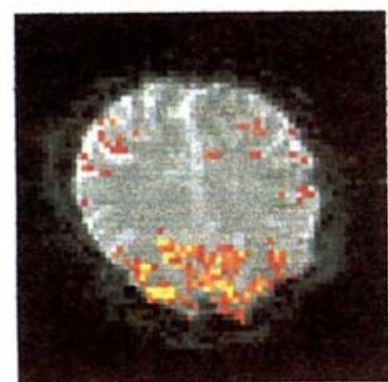

Fig. 9 Student $t$-statistic mapping of a visual stimulation fMRI. It was thresholded at $p<0.01$ (onetail).
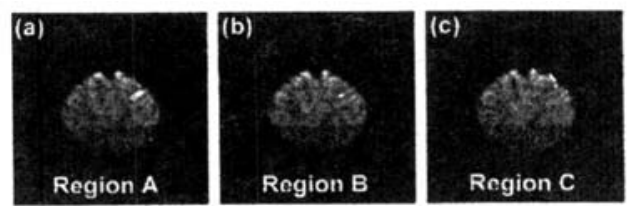

(d)

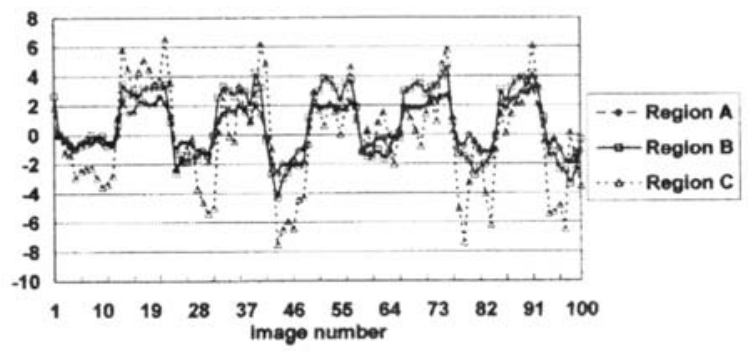

Fig. 10 Regions with different signal dynamics identified by the clustering analysis method cascading $K C N$ and FCM. Two of the regions $(a, b)$ are in the primary motor area and the other $(c)$ is in the premotor area.

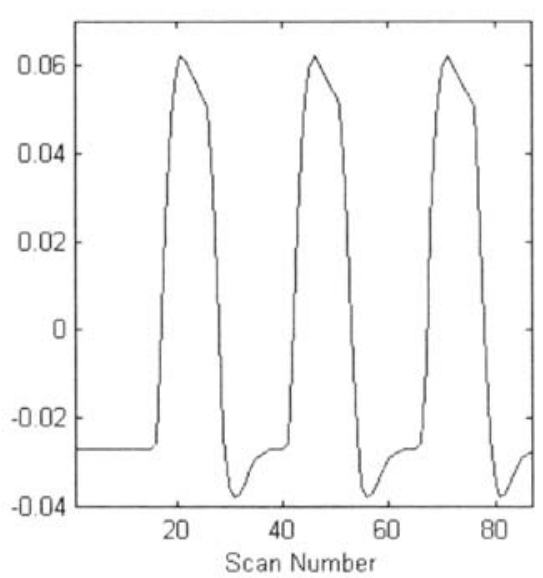

Fig. 8 (a) Idealized hemodynamic response function. (b) The postulated functional response waveform (reference waveform) after convolving the stimulus paradigm with the HRF shown in (a). 
dom chance. Using the following equation, cvery pixcl has an associated value of the test statistic.

$$
t=\frac{\frac{1}{N_{A}} \sum_{t=1}^{N_{A}} A_{(x, y)}(t)-\frac{1}{N_{B}} \sum_{i=1}^{N_{g}} R_{(x, y)}(t)}{\sqrt{\frac{S_{A}^{2}+S_{R}^{2}}{N_{A}+N_{B}}}}
$$

where $\mathrm{A}_{(\mathrm{x}, \mathrm{y})}(t)$ and $\mathrm{R}_{(\mathrm{x}, \mathrm{y})}(t)$ represent signal intensity of the activated state and the control (rest) state, respectively, at time $t$ and coordinate $(x, y) ; \mathrm{S}_{\mathrm{A}}{ }_{\mathrm{A}}$ is the variance of $A_{(x, y)}(t) ; S_{R}^{2}$ is the variance of $R_{(x, y)}(t)$; and $\mathrm{N}$ is the number of the time points for functional images. By mapping each of these values to the location corresponding to each pixel, a statistical parametric map can be constructed to provide a means of visualizing the spatial location, extent, and magnitude of brain activity (Fig. 9).

\subsection{General Linear Model}

The idea of general linear model (GLM) extends the simple two-condition model of $t$-test to simultaneously consider more parameters that could influence the observed data. In GLM, the signal of a pixel $y$ is modeled as a linear combination of $p$ experimental conditions:

$$
y_{i}=\beta_{0}+x_{i 1} \beta_{1}+\cdots+x_{i k} \beta_{k}+\cdots+x_{i p} \beta_{p}+\varepsilon_{i}
$$

where $y_{i}$ is the signal at time $i$ (i.e. $i$ th scan), $i=1, \ldots, N$; $\beta_{k}$ represents the influence of the experimental condition $k$ on $y_{i} ; \beta_{0}$ is the signal baseline; and $\varepsilon_{t}$ is the residual error, which is modeled as an identically independent Gaussian distribution of zero mean (iid). Expressed in matrix form, Eq. 6 becomes:

$$
\boldsymbol{y}=\mathbf{X} \boldsymbol{\beta}+\boldsymbol{\varepsilon}
$$

where $\mathbf{X}$ is also called the design matrix, $\beta$ is the parameter vector, and $\varepsilon$ is the error vector. The GLM will estimate the parameter vector that minimizes the error vector. To get statistical inference, $t$-statistic can be computed to evaluate the significance level of the cstimated parameters with respect to a weighted linear combination of the experimental conditions, called contrast.

\section{MODEL-FREE ANALYSIS}

\subsection{Independent Component Analysis}

ICA is a method for recovering underlying source signals from the linear mixtures of these signals. By minimizing the mutual information, it separates the mixed signal into statistically independent components[27]. One way to apply ICA to fMRI analysis is to find the spatially independent components in $\mathrm{MRI}$ data [28]. The reason is that brain areas activated by performing a psychomotor task should be unrelated to the brain areas whose signals are affected by artifacts, like physiological motion, head movement, etc. Each of these separate processes may be represented by one or more spatially independent components, each associated with a single time course of enhancement and/or suppression. Using this method, the task-related functional response can be separated from various noise sources without knowing the stimulus paradigm or making assumption about the response waveform. It has been shown that it is possible to identify and extract physiological activities related to cardiac pulsation and respiration[29] using such method.

\subsection{Temporal Clustering by Kohonen Cluster- ing Network and Fuzzy C-Means}

Another approach is to classify time signals of the brain into several patterns according to temporal similarity among these signals. Kohonen Clustering Network (KCN) is derived from Kohonen's selforganizing feature map[30]. It attempts to reduce the data dimension by minimizing the Euclidean distance between MR signal and cluster centroid. Based on competitive learning, it is very efficient in clustering large amount of data like fMRI, but it is easily influenced by factors such as initial condition, learning rate[31]. Fuzzy c-means (FCM) is another unsupervised clustering method that can be used to identify patterns with different temporal activities. Similarity is determined by a fuzzy membership function between each input waveform and cluster centroid $[32,33]$. Unlike $\mathrm{KCN}$, which forces each input to belong to one of the clusters, FCM measures the probability an input belongs to each cluster. Thus it provides better discriminability of ambiguous patterns. But its computational demand is very high and is not suitable for processing large amount of data. The pitfalls of both methods can be reconciled by taking $\mathrm{KCN}$ as the first stage in clustering and FCM as the second stage to reorganize the similar patterns. With this cascade architecture, we benefit from the efficiency of the KCN in terms of high computation power and low memory load for the computer as well as the optimization capability of FCM [31].

Since this method does not need to make any assumption about the signal, it is very useful in extracting the "actual" functional responses. This capability is especially important in analyzing data with complex signal dynamics, such as event-related Fmri [34] or study of early negative response [35]. Furthermore, compared with ICA, this method is more sensitive to activities with different temporal patterns. Thus it is very useful in detecting temporally varying artifacts, such as head motion or physiological noises, and activation signals with different intensity, phase lag, or waveform. For example, three different kinds of acti- 
vation patterns can be identified in motor cortex undergoing a writing task (Fig. 10) [36]. This provides us an approach to investigate the detailed underlying physiology of MR signal.

\section{SUMMARY}

Functional MRI can provide important new insights into the phenomenon of brain activity through improved temporal and spatial resolution as well as easiness and safety on repeated or prolonged examination. A revolutionary renewal on the knowledge of functional anatomy of the human brain based on the pre-fMRI technology is thus possible. However, the activity in the brain is so complex that the simplified model-dependent analysis methods may not be sufficient. Therefore, we have endeavored to establish datadriven tools and methodologies for fMRI. Unsupervised algorithms based on self-organized artificial neural network and fuzzy logic are used to solve the problem of biased result or misled interpretation frequently encountered in statistical methods depending on prior knowledge of imposed experimental paradigm or assumed pattern of brain response to activation. With these new analysis methods, more accurate activation regions and physiological dynamics can be identified that makes further investigation possible and easier.

\section{REFERENCES}

1. Ogawa S, Lee TM, Nayak AS, Glynn P. Oxygensensitive contrast in magnetic resonance image of rodent brain at high magnetic fields. Magn Reson Med 1990;14:68-78.

2. Ogawa S, Lee TM, Kay AR, Tank DW. Brain magnetic resonance imaging with contrast dependent on blood oxygenation. Proc Natl Acad Sci USA 1992;87:9868-72.

3. Kwong KK, Belliveau JW, Chesler DA, et al. Dynamic magnetic imaging of human brain activity during primary sensory stimulation. Proc Natl Acad Sci USA 1992;89:5675-9.

4. Belliveau JW, Kwong KK, Kennedy DN, et al. Magnetic resonance imaging mapping of brain function: human visual cortex. Invest Radiol 1992; 27:559.

5. Belliveau JW, Rosen BR, Kantor HL, et al. Functional cerebral imaging by susceptibility contrast NMR. Magn Reson Med 1990;14:538.

6. Belliveau JW, Kennedy DN, McKinstry RC et al: Funcitonal mapping of the human visual cortex by magnetic resonance imaging, Science 254:716-9, 1991.

7. Williams DS, Detre JA, Leigh JS, et al. Magnetic resonance of perfusion using spin inversion of arte- rial water. Proc Nall Acad Sci USA 1992;89:212.

8. Kim SG. Quantification of relative cerebral blood flow change by flow-sensitive alternating inversion recovery (FAIR) technique: application to functional mapping. Magn Reson Med 1995;34:293-301.

9. Kwong KK, Chesler DA, Weisskoff RM et al. MR perfusion studies with T1-weighted echo planar imaging. Magn Reson Med 1995;34:878-887.

10. Pauling L, Coryell CD. The magnetic properties and structure of hemoglobin, oxyhemoglobin and carbonmonoxyhemoglobin Proc. Natl. Acad. Sci. USA 1936; 22: 210-6.

11. Thulborn KWJ, Matthews $P$ et al. Oxygenation dependence of the transverse relaxation time of water protons in whole blood at high field. Biochem Biophys Acta 1982;714:265.

12. Ogawa $S$, Lee $T$. Magnetic resonance imaging of blood vessels at high fields: in vivo and in vitro measurements and image simulation. Magn Reson Med 1990;16:9.

13. Fox PT, Raichle ME. Focal physiological uncoupling of cerebral blood flow and oxidative mechanism during somatosensory stimulation in human subjects. Proc Natl Acad Sci USA 1986;83:1140-4.

14. Fox PT, Raichle ME, Mintun MA, Dence C. Nonoxidative glucose consumption during focal physiologic neural activity. Science 1988; 241:462-4.

15. Deyoe E, Bandettini P, Neitz J et al. Functional magnetic resonance imaging (fMRI) of the human brain. J Neurosci Methods 1994;54:171.

16. Woods R, Cherry S, Mazziotta J. Rapid automated algorithm for aligning and reslicing PET images. $J$ Comp Assist Tomogr 1992;16:620-633.

17. Friston KJ, Williams S, Howard R, Frackowiak RS, Turner R. Movement related effects in fMRI timeseries. Magn Reson Med 1996;35:346 -355.

18. Talairach P, Tournoux, J. A stereotactic coplanar atlas of the human brain. Thieme. Stuttgart, 1998.

19. Fox PT, Perlmutter JS, Raichle ME. A stcreotactic method of anatomical localization for position emission tomography. $J$ Comput Assist Tomogr 1985;9:141-153.

20. Sijbers J, den Dekker AJ, Van der Linden A, Verhoye TM, Van Dyck D. Adaptive anisotropic noise filtering for magnitude MR data. Magn Reson Imaging. 1999; 17:1533-9.

21. Chiu MJ, Lin CC, Chuang KH, Chen JH, Huang $\mathrm{KM}$. Tissuc segmentation-assisted analysis of $\mathrm{fMRI}$ for human motor response: an approach combining artificial neural network and fuzzy $\mathrm{C}$ means. $J$ Digit Imaging. 2001;14:38-47.

22. Bandettini PA, Jesmanowicz A, Wong EC, Hyde JS. Processing strategies for time-course data sets in functional MRI of the human brain. Magn Reson Med 1993;30:161-173.

23. Friston $K J$, Jezzard $P$, Tumer R. Analysis of functional MRI time-series. Human brain mapping 
1994;1:153-171.

24. Friston KJ, Holmes AP, Worsley KJ, Poline J-B, Frith CD, Frackowiak RSJ. Statistical Parametric Maps in Functional Imaging - A General Linear Approach. Human Brain Mapping 1995;2:189-210.

25. Liu Y, Gao J-H, Liu H-L, Fox PT. The temporal response of the brain after cating revealed by functional MRI. Nature 2000; 405:1058-62.

26. Sobel N, Prabhakaran V, Zhao Z, Demond JE, Glover GH, Sullivan EV, Gabrieli JD. Time course of odorant-induced activation in the human primary olfactory cortex. J. Neurophysiol. 2000;83:537-551.

27. Bell AJ, Sejnowski TJ. An informationmaximization approach to blind separation and blind deconvolution. Neural Comput 1995;7:1129 1159.

28. McKeown MJ, Makeig S, Brown GG, Jung T-P, Kindermann SS, Bell AJ, Sejnowski TJ. Analysis of fMRI data by decomposition into independent spatial components. Hum Brain Mapp 1998;6:160188.

29. Chuang $\mathrm{KH}$, Chen JH. Independent component analysis in the detection and correction of physiological artifacts in fMRI. Conf Human Brain Mapping, Brighton, UK. 2001.

30. Fischer H, Hennig J. Neural network-based analysis of MR time series. Magn Reson Med. 1999;41:124-31.

31. Chuang KH, Chiu MJ, Lin CC, Chen JH. Model- free functional MRI analysis using Kohonen clustering neural network and fuzzy c-means. IEEE trans Med Imaging 1999;18:1117-28.

32. Baumgartner R, Scarth G, Teichtmeister C, Somorjai R, Moser E. Fuzzy clustering of gradient-echo functional MRI in the human visual cortex. Part I: reproducibility. J Magn Reson Imaging. 1997;7:1094-101.

33. Golay X, Kollias S, Stoll G, Meier D, Valavanis A, Boesiger P. A new correlation-based fuzzy logic clustering algorithm for fMRI. Magn Reson Med. 1998;40:249-60.

34. Buckner RL, Bandettini PA, O'Craven KM, Savoy RL, Petersen SE, Raichle ME, Rosen BR. Detection of cortical activation during averaged single trials of a cognitive task using functional magnetic resonance imaging. Proc Natl Acad Sci USA 1996;93:14878-14883.

35. Hu X, Le TH, Ugurbil K. Evaluation of the carly response in fMRI in individual subjects using short stimulus duration. Magn. Reson. Med. 1997;37: 877-884.

36. Chuang KH, Chiu MJ, Lin CC, Chen JH. Finding different physiological signal sources of fMRI data using paradigm-free Kohonen clustering network and fuzzy c-means. Proc European Soc. Magn Reson Med Biology (ESMRMB), Geneva, Switzerland, 1998. 